\title{
Flow of a Williamson Fluid over a Stretching Sheet Containing Nanoparticles
}

\author{
Farah Deba, Abdul Rehman, Zahida Khan, Saleem Iqbal, Naveed Sheikh \\ Department of Mathematics, University of Balochistan, Quetta, Pakistan \\ Email address: \\ farah.math1000@gmail.com (F. Deba) \\ To cite this article: \\ Farah Deba, Abdul Rehman, Zahida Khan, Saleem Iqbal, Naveed Sheikh. Flow of a Williamson Fluid over a Stretching Sheet Containing \\ Nanoparticles. International Journal of Systems Engineering. Vol. 5, No. 1, 2021, pp. 13-17. doi: 10.11648/j.ijse.20210501.12
}

Received: November 18, 2020; Accepted: April 14, 2021; Published: April 30, 2021

\begin{abstract}
In this article, an attempt has been made to study the behavior of non-Newtonian viscoelastic model of a Williamson fluid flow problem containing nanoparticles and is assumed to be flowing over a stretching sheet stretched along its surface in both directions with the same constant surface stretching velocity. The boundary layer governing equations of the conservation of mass, conservation of linear momentum and the energy are first modeled into a set of nonlinear coupled partial differential equations along with the appropriate boundary conditions. A numerical study of impact of nanofluid flow over a stretching sheet is tabulated. The basic equations of Williamson fluid are modeled with the help of Navier-Stokes equations for momentum and heat transfer. With the assistance of appropriate similarity transformations these pair of PEDs is transformed into up-to-date system of coupled nonlinear ODEs. These transformed systems of equations are evaluated numerically with the assistance of shooting method using forth order. The dominant physical properties for system of equations of the model that is wall shear stress, and the coefficient of skin friction are acquired. The behavior of nondimensional velocity and thermal flow profiles are discussed for the important involved dimensionless parameters like the Williamson fluid parameter and the nanoparticles volume fraction through tables and graphs.
\end{abstract}

Keywords: Boundary Layer Flow, Williamson Fluid, Nanoparticles, Stretching Sheet

\section{Introduction}

In engineering and industrial processes, the impact of nonNewtonian fluids cannot be hided, and the field is fascinating for researchers. Various fluid models have been projected to define the behavior of pseudoplastic fluids and one of the prevalent fluids under this category is Williamson fluid. The most prominent application of this fluid is in biological engineering; where it is used in estimating heat and mass transfer in blood vessels and analyzed diffusion of nutrients in human blood. The concept of nanofluids opens new gateway and has vast applications in the area of physics, chemical engineering, metallurgical engineering and heat transfer. Williamson [1] has studied and projected some models to discuss the flow of pseudoplastic. Dapra and Scarpi [2] analyzed the perturbation solutions for boundary layer flow of Williamson fluid. The concept of nanofluids was derived by Chio [3]. Crane [4] considered the boundary layer flow over a stretching sheet. Nadeem et al. [5] studied Williamson fluid model for Peristaltic flows. Vasudev et al.
[6] considered Williamson fluid model for the boundary layer flow and heat transfer of peristaltic pumping through some porous medium. Cramer et al. [7] compared the experimental data of particle suspensions polymer solutions with the Williamson fluid model. Khan and Pop [8] investigated the boundary layer flow of nanofluid flow over a stretching sheet. Their work was extended by Makinde and Aziz [9] for the flow with convective boundary conditions. Sakiadis [10] formulated boundary layer flow problem for continuously moving surface. Tsou et al. [11] studied the heat transmission of boundary layer flow over a continuous surface. The problem of suction/injection flow and transfer of heat over a stretching surface was discussed by Erickson et al. [12]. Few other related studies are cited in [13-16].

The main intention of the study is to deliver a numerical solution for the underlying model based on boundary layer flow in case of Williamson fluid flowing over a starching sheet. The numerical results are presented both graphically 
and in tabular form.

\section{Mathematical Formulation}

Consider the Cartesian coordinates in two-dimensional plane [17-20] that is $x$ and $y$ - axis and suppose steady incompressible flow for Williamson fluid past a stretching sheet. Additionally, the impact of nanoparticles is saturated under these conditions, and sheet for this problem is specifically stretched with the plane $y=0$. Assume that the flow of liquid is flowing towards the positive side of $y$ - axis $y>0$. In our problem we suppose that the sheet is stretched along with linear velocity represented as $u_{w}(x)=a x$, in the equation for velocity the constant coefficient of $x$ that is a is always positive $a>0$ and evaluate the stretching surface along with $x$ - axis. Two forces are applied for our problem along with $x$ - axis but these forces are equal in magnitude and opposite in direction to invoke the stretching in sheet but keeping the origin stick at zero. Here $T_{w}$ represents the fixed temperature observe at the surface of sheet and the other type of temperature which is known as ambient temperature is usually represents as $T_{\infty}$. The flow is produced due to the linear stretching in the sheet. In the lack of a body force of the system, the components of governing equations of the model are mathematically observed as [21-24]:

$$
\begin{gathered}
\frac{\partial u}{\partial x}+\frac{\partial v}{\partial y}=0 \\
u \frac{\partial u}{\partial x}+v \frac{\partial y}{\partial y}=\frac{\mu_{n f}}{\rho_{n f}} \frac{\partial^{2} u}{\partial y^{2}}+\sqrt{2} v \Gamma \frac{\partial u}{\partial y} \frac{\partial^{2} u}{\partial y^{2}} \\
u \frac{\partial u}{\partial x}+v \frac{\partial y}{\partial y}=\alpha_{n f} \frac{\partial^{2} T}{\partial y^{2}}
\end{gathered}
$$

The components of the velocity are taken as $u(x, y)$ and $v(x, y)$ along the direction of flow as well as orthogonal to the direction of flow respectively, temperature is denoted as $T$, here thermal diffusivity of nanofluid is represented as $\alpha_{n f}$. Moreover, the effective density of the nanofluid is represented as $\rho_{n f}$ and effective viscosity of the nanofluid is denoted as $\mu_{n f}$, these terms are introduced by Oztop and Abu-Nada

$$
\begin{gathered}
\mu_{n f}=\frac{\mu_{f}}{(1-\phi)^{2.5}}, \quad \rho_{n f}=(1-\phi) \rho_{f}+\phi \rho_{s} \\
\alpha_{n f}=\frac{k_{n f}}{\left(\rho C_{p}\right)_{n f}}
\end{gathered}
$$

Here, $\rho_{f}$ is used to represents the density of base fluid of the model along with this $\rho_{s}$ is to represents nanoparticles' density and $\phi$ represents for parameter for volume fraction of nanofluid or solid volume of that fluid, $k_{n f}$ shows the effective thermal conductivity present in the nanofluid [2526]. The resulting boundary conditions of the problem are listed in the form:

$$
\begin{gathered}
u=U_{w}=B x ; v=0 ; T=T_{w} \text { at } y=0 \\
u \rightarrow 0, T \rightarrow \infty \text { as } y \rightarrow \infty
\end{gathered}
$$

Where kinematic viscosity is usually represented as $v$, also the velocity of the system which is observed as at wall velocity is denoted as $U_{w}$ and parameter for stretching is always positive represented as $B>0$. Introducing the following similarity transformations:

$$
\begin{gathered}
u=B x f^{\prime}(\eta), v=-\sqrt{B v} f(\eta), \eta=\sqrt{\frac{B}{v} y} \\
\theta(\eta)=\frac{T-T_{\infty}}{T_{w}-T_{\infty}}
\end{gathered}
$$

Making use of transformations (7), equation (2) and (3) takes the form:

$$
\begin{gathered}
\frac{1}{(1-\phi)^{2.5}\left[(1-\phi)+\phi\left(\rho_{s} / \rho_{f}\right)\right]} f^{\prime \prime \prime}-f^{\prime 2}+f f^{\prime \prime}+\lambda f^{\prime \prime} f^{\prime \prime \prime}=0 \\
\frac{k_{n f} / k_{k}}{\left[(1-\phi)+\phi\left(\left(\rho C_{p}\right)_{s} /\left(\rho C_{p}\right)_{f}\right)\right]} \frac{1}{\operatorname{Pr}} \theta^{\prime \prime}+f \theta^{\prime}=0
\end{gathered}
$$

Boundary conditions of the problem are observed as:

$$
\begin{gathered}
f=0, \theta=1, f^{\prime}=1 \text { at } \eta=0 \\
f^{\prime} \rightarrow 0, \theta \rightarrow 0 \text { as } \eta \rightarrow \infty
\end{gathered}
$$

Where $\lambda$ corresponds to the dimensionless parameter known as Williamson parameter and mathematically defined as follows:

$$
\lambda=\Gamma x \sqrt{\frac{2 B^{2}}{v}}
$$

For $\lambda=0$, equation (11) transferred into boundary layer equations which is in the classical form for viscous flow of liquid, $\tau_{w}$ represents the shear stress which is related to wall and observed as physical quantity of underlying problem and the quantity denoted as $c_{f}$ known as the coefficient of skin fraction. After applied the approximations for boundary layer is denoted as, $\tau_{w}$ is given by [27-30].

$$
\tau_{w}=\left.\mu_{o}\left[\frac{\partial u}{\partial y}+\frac{\Gamma}{\sqrt{2}}\left(\frac{\partial u}{\partial y}\right)^{2}\right]\right|_{y=0}
$$


The coefficient of skin friction for the given problem is represented in mathematical form as:

$$
c_{f}=\frac{\tau_{w}}{\rho U_{w}^{2}}
$$

In dimensionless form for the coefficient for skin fraction for the problem is represented in mathematical form as:

$$
\sqrt{\operatorname{Re} c_{f}}=\left[\left(f^{\prime \prime}+\frac{\lambda}{2} f^{\prime \prime 2}\right)\right]_{\eta=0}
$$

Where $\operatorname{Re}=\frac{B x^{2}}{v}$ is the Reynolds number. Also, we have

$$
\operatorname{Pr}=\frac{v_{f}}{\alpha_{f}}
$$

\section{Numerical Solution}

With the help of shooting method, we can solve BVP problem numerically by taking equations (10-11) which are ordinary differential equations of non-linear type along with desired boundary condition according to the problem given in equations (12-13). In solution process take equations (10-11) along with boundary conditions in (12-13) and first step is to reduce these equations into first order equations and then implement initial guess to run the iterative process of shooting method to reach at the desired approximate solution [31-34].

\section{Results and Discussion}

The acquire pair of non-linear partial differential equations is transformed from similarity transformation into ODEs along with suitable boundary conditions is numerically solved with help shooting technique by the assistance of Runge-Kutta method [35-37].

Now implementing shooting method iterative process in MATLAB coding to approach the approximate solution and based on that we work out for satisfactory IVP that gives the appropriate answer of solution for initially determine BVP for the fluid model and discuss the solution with help of graphical representation and tabular form [38-40].

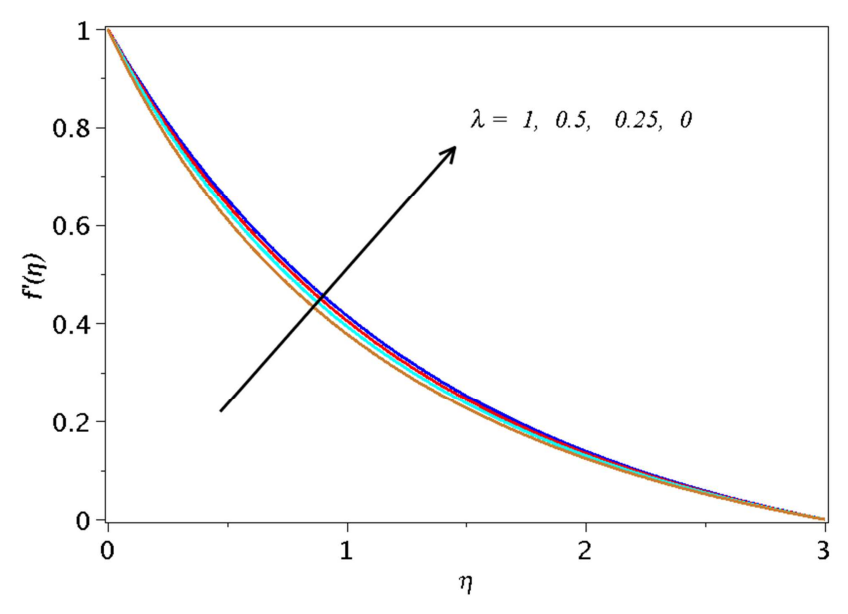

Figure 1. Velocity gradient for different $\lambda$.

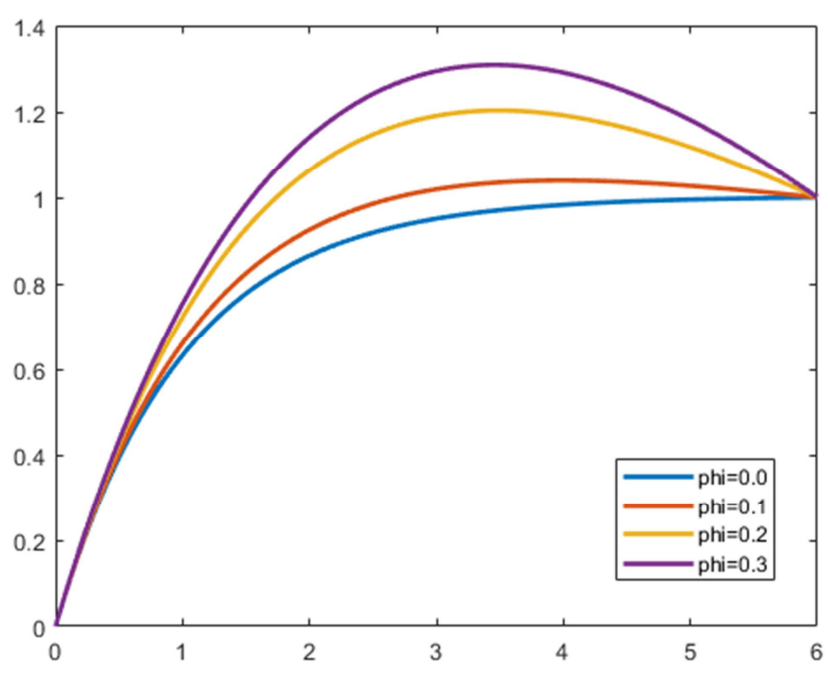

Figure 2. Velocity profile for different $\phi$.

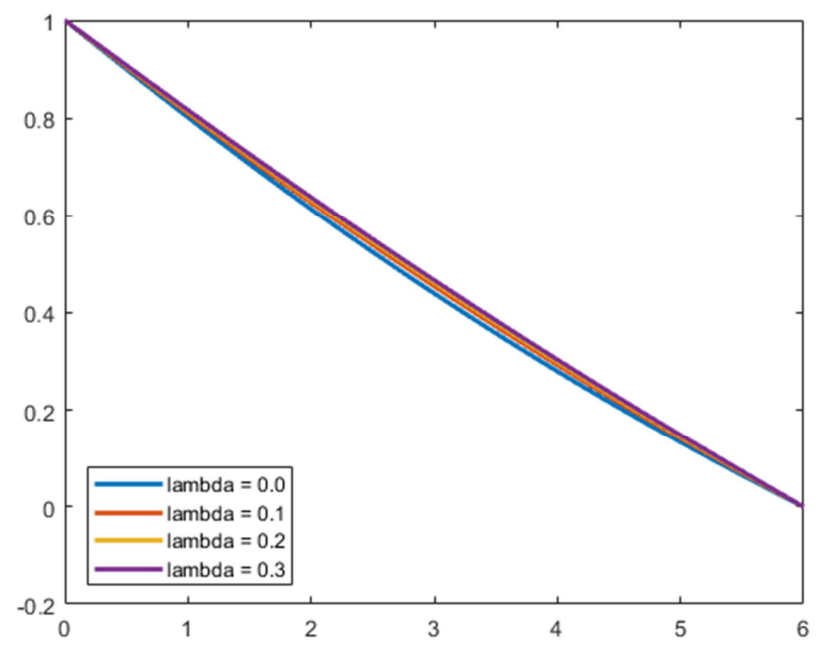

Figure 3. Temperature profile for different $\lambda$. 


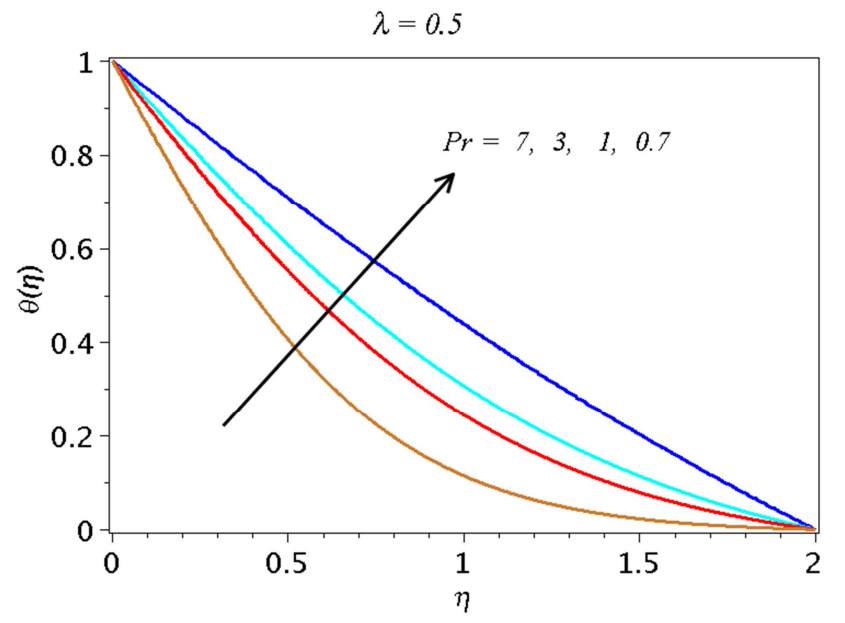

Figure 4. Temperature profile for different $\mathrm{Pr}$.

Table 1. Velocity profile for different $\phi$

\begin{tabular}{ll}
\hline $\boldsymbol{\phi}$ & $\boldsymbol{f}$ \\
\hline 0.0 & 0.0100 \\
0.1 & 0.1071 \\
0.2 & 0.2057 \\
0.3 & 0.2952 \\
\hline
\end{tabular}

Table 2. Temperature profile for different $\lambda$.

\begin{tabular}{ll}
\hline$\lambda$ & $\boldsymbol{\theta}$ \\
\hline 0.0 & 0.9980 \\
0.1 & 0.9753 \\
0.2 & 0.9601 \\
0.3 & 0.9417 \\
\hline
\end{tabular}

\section{Conclusions}

In this present paper, we have considered model of Williamson fluid containing nanoparticles and analyzed flow in two-dimensional on that model over stretching sheet. The system of equations of the model for Williamson fluid are evolved with the aid of Navier-Stoke equations for momentum and heat and with help suitable similarly transformations these derived modeled equations in the shape of PDE are settled into ODEs. Now these transformed equations are solved numerically with the aid of Runge - Kutta (RK) technique with shooting method. The impact of various types of parameters like Reynolds number, coefficient of skin friction and Williamson parameter are attained and the convergence of the solution is observed. At the end graphical behavior of solutions are also discussed in detail.

\section{References}

[1] Williamson, R. V., The flow of pseudoplastic materials. Industrial \& Engineering Chemistry Research, 21, No. 11, 1108 (1929).

[2] Dapra and Scarpi, G., Perturbation solution for pulsatile flow of a non-Newtonian Williamson fluid in a rock fracture. International Journal of Rock Mechanics and Mining Sciences, 44, 271 (2007).
[3] S. U. S. Choi, "Enhancing thermal conductivity of fluids with nanoparticle," in Developments and Applications of NonNewtonian Flows, D. A. Siginer and H. P. Wang, Eds., vol. 231, pp. 99-105, ASME, New York, NY, USA. (1995).

[4] Crane LJ. Flow past a stretching plate. Zeitschrift für angewandte Mathematik und Physik (Z AMP). 197 0; 21(4): 645- 647.

[5] Nadeem, S. and Akbar, N. S., Numerical solutions of peristaltic flow of Williamson fluid with radially varying MHD in an endoscope. International Journal for Numerical Methods in Fluids, 66, No. 2, 212 (2010).

[6] Vasudev, C., Rao, U. R., Reddy, M. V. S. and Rao, G. P., Peristaltic pumping of Williamson fluid through a porous medium in a horizontal channel with heat transfer. American Journal of Scientific and Industrial Research, 1, No. 3, 656 (2010).

[7] Cramer, S. D. and Marchello, J. M., Numerical evaluation of models describing non-Newtonian behavior. American Institute of Chemical Engineers Journal, 14, 980 (1968).

[8] Khan WA, Pop I. Boundary-layer flow of a nanofluid past a stretching sheet. International Journal of Heat and Mass Transfer. (2010) 53 (11): 2477-2483.

[9] Makinde OD, Aziz A. Boundary layer flow of a nanofluid past a stretching sheet with a convective boundary condition. International Journal of Thermal Sciences. 2011; 50 (7): 13261332.

[10] Sakiadis, B. C., Boundary layer behavior on continuous solid flat surfaces. American Institute of Chemical Engineers Journal, 7, 26 (1961).

[11] Tsou, F. K., Sparrow, E. M. and Goldstein, R. J., Flow And heat transfer in the boundary layer on a continuous moving surface. International Journal of Heat and Mass Transfer, 10, 219 (1967).

[12] Erickson, L. E., Fan, L. T. and Fox, V. G., Heat and mass transfer in the laminar boundary layer flow of a moving flat surface with constant surface velocity and temperature focusing on the effects of suction/injection. Industrial \& Engineering Chemistry Research, 5, 19 (1966).

[13] Gupta, P. S. and Gupta, A. S., Heat and mass transfer on a stretching sheet with suction or blowing. Canadian Journal of Chemical Engineering, 55, No. 6, 744 (1977).

[14] Ishak, A., Nazar, R. and Pop, I., Heat Transfer over a stretching surface with variable heat flux in micropolar fluids. Physics Letters A, 372, No. 5, 559 (2008).

[15] Nadeem, S., Hussain, A. and Khan, M., HAM solutions for boundary layer flow in the region of the stagnation point towards a stretching sheet. Communications in Nonlinear Science and Numerical Simulation, 15, No. 3, 475 (2010).

[16] S. Nadeem, Abdul Rehman, K. Vajravelu, Jinho Lee, Changhoon Lee, Axisymmetric stagnation flow of a micropolar nanofluid in a moving cylinder, Mathematical Problems in Engineering, Volume 2012, Article ID 378259.

[17] Abdul Rehman, S. Nadeem, Mixed convection heat transfer in micropolar nanofluid over a vertical slender cylinder, Chin. Phy. Lett. 29 (12) (2012) 124701-5.

[18] S. Nadeem, Abdul Rehman, Changhoon Lee, Jinho Lee, Boundary layer flow of second grade fluid in a cylinder with heat transfer, Mathematical Problems in Engineering, Volume 2012, Article ID 640289. 
[19] S. Nadeem, Abdul Rehman, Mohamed Ali, The boundary layer flow and heat transfer of a nanofluid over a vertical slender cylinder, J. NanoEngineering and NanoSystems (2012) $1-9$.

[20] S. Nadeem, Abdul Rehman, Axisymmetric stagnation flow of a nanofluid in a moving cylinder, Comp. Math. Mod. 24 (2) (2013) 293-306.

[21] Abdul Rehman, S. Nadeem, M. Y. Malik, Stagnation flow of couple stress nanofluid over an exponentially stretching sheet through a porous medium, J. Power Tech. 93(2) (2013) 122-132.

[22] Abdul Rehman, S. Nadeem, M. Y. Malik, Boundary layer stagnation-point flow of a third-grade fluid over an exponentially stretching sheet, Braz. J. Che. Eng. 30 (3) (2013) 611-618.

[23] Abdul Rehman, S. Nadeem, Heat transfer analysis of the boundary layer flow over a vertical exponentially stretching cylinder, Global J. Sci. Fron. Res. 13(11) (2013) 73-85.

[24] M. Y. Malik, M. Naseer, S. Nadeem, Abdul Rehman, The boundary layer flow of Casson nanofluid over a vertical exponentially stretching cylinder, Appl. NanoSci. DOI: $10.1007 / \mathrm{s} 13204-012-0267-0$

[25] Abdul Rehman, S. Nadeem, S. Iqbal, M. Y. Malik, M. Naseer, Nanoparticle effect over the boundary layer flow over an exponentially stretching cylinder, J. NanoEngineering and NanoSystems (2014) 1-6.

[26] M. Y. Malik, M. Naseer, S. Nadeem, Abdul Rehman, The boundary layer flow of hyperbolic tangent fluid over a vertical exponentially stretching cylinder, Alexandria Eng. J., 53 (2014) 747-750.

[27] M. Y. Malik, M. Naseer, Abdul Rehman, Numerical study of convective heat transfer on the Power Law fluid over a vertical exponentially stretching cylinder, App Comp Math, 4(5), (2015) 346-350.

[28] Abdul Rehman, R. Bazai, S. Achakzai, S. Iqbal, M. Naseer, Boundary Layer Flow and Heat Transfer of Micropolar Fluid over a Vertical Exponentially Stretched Cylinder, App Comp Math, 4(6) (2015) 424-430.

[29] Abdul Rehman, G. Farooq, I. Ahmed, M. Naseer, M. Zulfiqar, Boundary Layer Stagnation-Point Flow of Second Grade Fluid over an Exponentially Stretching Sheet, American J App Math Stat, 3(6) (2015) 211-219.

[30] Abdul Rehmana, S. Achakzai, S. Nadeem, S. Iqbal, Stagnation point flow of Eyring Powell fluid in a vertical cylinder with heat transfer, Journal of Power Technologies 96 (1) (2016) $57-62$.
[31] Abdul Rehman, Saleem Iqbal, Syed Mohsin Raza, Axisymmetric Stagnation Flow of a Micropolar Fluid in a Moving Cylinder: An Analytical Solution, Fluid Mechanics, 2(1) (2016) 1-7.

[32] Naheeda Iftikhar, Abdul Rehman, Peristaltic flow of an Eyring Prandtl fluid in a diverging tube with heat and mass transfer, International Journal of Heat and Mass Transfer 111 (2017) 667-676.

[33] Abdul Rehman, Naveed Sheikh, Boundary Layer StagnationPoint Flow of Micropolar Fluid over an Exponentially Stretching Sheet, International Journal of Fluid Mechanics \& Thermal Sciences, 2017; 3 (3): 25-31.

[34] Haroon Rasheed, Abdul Rehman, Naveed Sheikh, Saleem Iqbal, MHD Boundary Layer Flow of Nanofluid over a Continuously Moving Stretching Surface, Applied and Computational Mathematics, 2017; 6 (6): 265-270.

[35] Najeeb Alam Khan, Umair Bin Saeed, Faqiha Sultan, Saif Ullah, and Abdul Rehman, Study of velocity and temperature distributions in boundary layer flow of fourth grade fluid over an exponential stretching sheet, AIP ADVANCES 8, 025011 (2018).

[36] Naheeda Iftikhar, Abdul Rehman, Hina Sadaf, Muhammad Najam Khan, Impact of wall properties on the peristaltic flow of $\mathrm{Cu}$-water nano fluid in a non-uniform inclined tube, International Journal of Heat and Mass Transfer 125 (2018) 772-779.

[37] Naheeda Iftikhar, Abdul Rehman and Muhammad Najam Khan, Features of Convective heat transfer on MHD peristaltic movement of Williamson fluid with the presence of Joule heating, IOP Conf. Series: Materials Science and Engineering 414 (2018) 012010.

[38] Amina Panezai, Abdul Rehman, Naveed Sheikh, Saleem Iqbal, Israr Ahmed, Manzoor Iqbal, Muhammad Zulfiqar, Mixed Convective Magnetohydrodynamic Heat Transfer Flow of Williamson Fluid Over a Porous Wedge, American Journal of Mathematical and Computer Modelling, 4(3) (2019) 66-73.

[39] N. Ambreen, A. Rehman, N. Sheikh, S. Iqbal, M. Zulfqar, Boundary-Layer Flow and Heat Transfer over a Rotating Porous Disk in a Non-Newtonian Williamson Nanofluid, Indian Journal of Science and Technology, 12(38) (2019) 1-8.

[40] Naheeda Iftikhar, Abdul Rehman, Hina Sadaf, and Saleem Iqbal, Study of $\mathrm{Al}_{2} \mathrm{O}_{3}$ /copper-water nanoparticle shape, slip effects, and heat transfer on steady physiological delivery of MHD hybrid nanofluid, Canadian Journal of Physics, 97 (12) (2019) 1239-1252. 